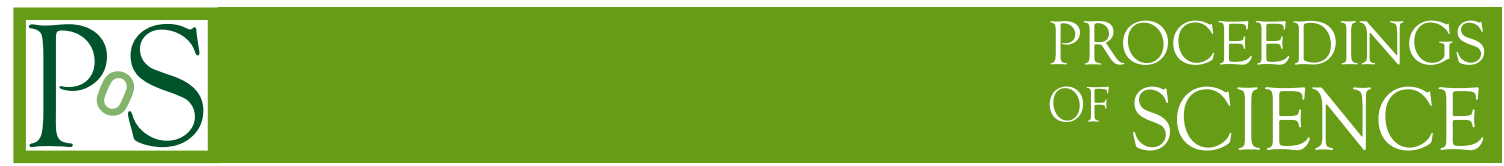

\title{
Effective theories of electroweak symmetry breaking
}

\author{
Gino Isidori*广 \\ INFN, Laboratori Nazionali di Frascati, Via E. Fermi 40 I-00044 Frascati, Italy \\ Institute for Advanced Study, T. U. München, Arcisstraße 21, D-80333 München, Germany \\ E-mail: Gino.Isidorielnf.infn.it
}

\begin{abstract}
We present a brief review of recent attempts to construct effective theories to describe the breaking of the electroweak symmetry in extensions of the Standard Model with new strongly interacting dynamics around the TeV scale. Particular attention is devoted to the analogies of Higgsless models with Chiral Perturbation Theory (the low-energy limit of QCD).
\end{abstract}

6th International Workshop on Chiral Dynamics, CD09

July 6-10, 2009

Bern, Switzerland

\footnotetext{
*Speaker.

${ }^{\dagger}$ Work supported by the EU under contract MTRN-CT-2006-035482.
} 


\section{Introduction}

Despite the impressive phenomenological success of the Standard Model (SM), our understanding of one of its key ingredients, namely the spontaneous breaking of the $S U(2)_{L} \times U(1)_{Y}$ gauge symmetry, is still very poor. We have very solid evidences that $S U(2)_{L} \times U(1)_{Y}$ is a local symmetry of the underlying theory, and that this symmetry is only spontaneously broken. However, the fact that this breaking is induced by the non-trivial vacuum expectation value of a single $S U(2)_{L}$ scalar field (the Higgs field), is far from being clearly established.

A fundamental Higgs boson is certainly the most economical way to explain the $S U(2)_{L} \times$ $U(1)_{Y} \rightarrow U(1)_{Q}$ breaking, and a light Higgs mass $\left(m_{h} \approx 100 \mathrm{GeV}\right)$ is also an efficient way to account for all the existing electroweak precision tests. However, on the one hand we have no direct evidences of the physical Higgs boson. On the other hand, the strong sensitivity of $m_{h}$ to short-distance scales poses a serious naturalness problem to the theory.

Several alternative symmetry-breaking mechanisms have been proposed in the literature, from Higgsless theories, to models with composite or partially-composite Higgs bosons. As we will discuss in the following, the low-energy features of all these models are universal and can be described in general terms using appropriate effective theory approaches.

\section{A closer look to the Standard Higgs sector}

Before starting the discussion of alternative scenarios, it is convenient to give a closer look to the standard Higgs mechanism. The symmetry-breaking part of the SM Lagrangian can be written as follows

$$
\begin{aligned}
\mathscr{L}_{\text {S.B. }}^{\mathrm{SM}} & =\mathscr{L}_{\text {Higgs }}+\mathscr{L}_{\text {Yukawa }}, \\
\mathscr{L}_{\text {Higgs }} & =\frac{1}{4} \operatorname{Tr}\left(D_{\mu} H^{\dagger} D_{\mu} H\right)+\frac{\mu^{2}}{4} \operatorname{Tr}\left(H^{\dagger} H\right)-\frac{\lambda}{16}\left[\operatorname{Tr}\left(H^{\dagger} H\right)\right]^{2} .
\end{aligned}
$$

Here we have expressed the Higgs field in terms of the $2 \times 2$ matrix $H$, defined by $H=\left(i \sigma_{2} \phi^{*}, \phi\right)$, in terms of the familiar $S U(2)_{L}$ complex doublet $\phi$ :

$$
\begin{aligned}
& \phi=\left(\begin{array}{c}
\phi_{1}+i \phi_{2} \\
\phi_{3}+i \phi_{4}
\end{array}\right), \quad H=\left(i \sigma_{2} \phi^{*}, \phi\right), \\
& D_{\mu} H=\partial_{\mu} H-i g T_{a} W_{\mu}^{a} H+i g^{\prime} H T_{3} B_{\mu}, \quad T_{a}=\frac{1}{2} \sigma^{a} .
\end{aligned}
$$

This notation has the advantage of making manifest the invariance of the SM Higgs potential under the global $S U(2)_{L} \times S U(2)_{R}$ symmetry, defined by $H \rightarrow g_{L} H g_{R}^{\dagger}$. This global symmetry is spontaneously broken into the diagonal subgroup $S U(2)_{L+R}$ (often known as custodial symmetry) by the Higgs vacuum expectation value: $\langle H\rangle=v \times I$, which implies the tree-level relations

$$
m_{W}^{2}=\frac{g^{2} v^{2}}{4}, \quad m_{Z}^{2}=\frac{m_{W}^{2}}{\cos ^{2} \theta_{W}},
$$

for $W$ and $Z$ boson masses $\left(\cos ^{2} \theta_{W}=g^{2} /\left(g^{2}+g^{\prime 2}\right),\left.v^{2}\right|_{\text {tree }}=\mu^{2} / \lambda\right)$. 
As shown by the expression of the covariant derivative in (2.4), the $S U(2)_{L}$ subgroup of this global symmetry is fully gauged, while only the $T_{3}$ component of $S U(2)_{R}$ is gauged, giving rise to an explicit breaking of the custodial symmetry beyond the tree-level. More precisely, $T_{3}^{R}$ allows to write the generator of the $U(1)_{Y}$ gauge group as

$$
Y=T_{3}^{R}+\frac{1}{2}(B-L)
$$

where $B$ and $L$ are the barion- and lepton-number generators (which appear in the covariant derivatives acting on the fermion fields). An additional source of breaking of the custodial symmetry is present in the Yukawa interaction, due to the difference between up- and down-quark Yukawa couplings:

$$
\mathscr{L}_{\text {Yukawa }}^{\text {quark }}=\frac{1}{2}\left(Y_{U}^{i}+Y_{D}^{i}\right) \bar{Q}_{L}^{i} H Q_{R}^{j}+\left(Y_{U}^{i}-Y_{D}^{i}\right) \bar{Q}_{L}^{i} H T_{3} Q_{R}^{j}+\text { h.c., } \quad Q_{L(R)}^{i}=\left(\begin{array}{c}
u_{L(R)}^{i} \\
d_{L(R)}^{i}
\end{array}\right) .
$$

All the physical parameters appearing in $\mathscr{L}_{\mathrm{S} \text {.B. }}^{\mathrm{SM}}$ have been determined with high accuracy but for the combination controlling the mass of the physical Higgs boson: $m_{h}=2 \mu^{2}=2 \lambda v^{2}$, at the tree level. In other words, we have a good determination of the ground state determined by $\mathscr{L}_{\mathrm{S} \text {.B. }}^{\mathrm{SM}}$. but only a limited knowledge of the dynamics behind the symmetry breaking mechanism.

A non-trivial sensitivity to the Higgs mechanism is obtained from the Electroweak Precision Observables (EPWO), and in particular by the two-point effective couplings controlling masses and wave-functions of the massive gauge-bosons,

$$
S=\left.\frac{16 \pi}{g g^{\prime}} \frac{\partial}{\partial q^{2}} \mathscr{A}\left(W_{3} \rightarrow B\right)\right|_{q^{2}=0} \quad \text { and } \quad T=\frac{1}{\alpha}\left(\frac{m_{W}^{2}}{m_{Z}^{2} \cos ^{2} \theta_{W}}-1\right) .
$$

Within the SM these two effective couplings can be predicted unambiguously in terms of the free parameters of $\mathscr{L}_{\mathrm{S} . \mathrm{B}}^{\mathrm{SM}}$ (including $m_{h}$ ), while experimental data allows us to determine them independently of any assumption on $m_{h}$. The comparison of data vs. theory (including all the relevant quantum corrections) is shown in Fig. 1. From this plot it is clear that a light Higgs boson is an economical and consistent way to accommodate existing data. However, it must be stressed that this conclusion holds under the hypothesis of an heavy cut-off for the SM viewed as an effective theory.

If we extend the theory to include new degrees degrees of freedom above a cut-off scale $\Lambda>v$ $(v \approx 246 \mathrm{GeV})$, we can view $\mathscr{L}^{\mathrm{SM}}$ as the low-energy limit of an effective Lagrangian, with an infinite tower of higher-dimensional operators suppressed by inverse powers of $\Lambda$. In this perspective, we should expect corrections to $S$ and $T$ by operators of the from

$$
Q_{S}=g g^{\prime} \operatorname{Tr}\left(H T_{3} B_{\mu \nu} H^{\dagger} T_{a} W_{\mu \nu}^{a}\right), \quad Q_{T}=e^{2}\left[\operatorname{Tr}\left(T_{3} H^{\dagger} D_{\mu} H\right)\right]^{2},
$$

appearing in the dimension-six part of the Lagrangian, $\mathscr{L}_{d=6}=\left(c_{T} / \Lambda^{2}\right) Q_{T}+\left(c_{S} / \Lambda^{2}\right) Q_{S}+\ldots$, namely

$$
\Delta S=-c_{S} \frac{16 \pi v^{2}}{\Lambda^{2}}, \quad \Delta T=c_{T} \frac{8 \pi v^{2}}{\Lambda^{2}} .
$$

Assuming $c_{S, T}=\mathscr{O}(1)$, as expected by naïve dimensional analysis, we conclude that the indication of a light Higgs $\left(m_{h} \lesssim 200 \mathrm{GeV}\right)$ holds for $\Lambda \gtrsim 4 \mathrm{GeV}$. 


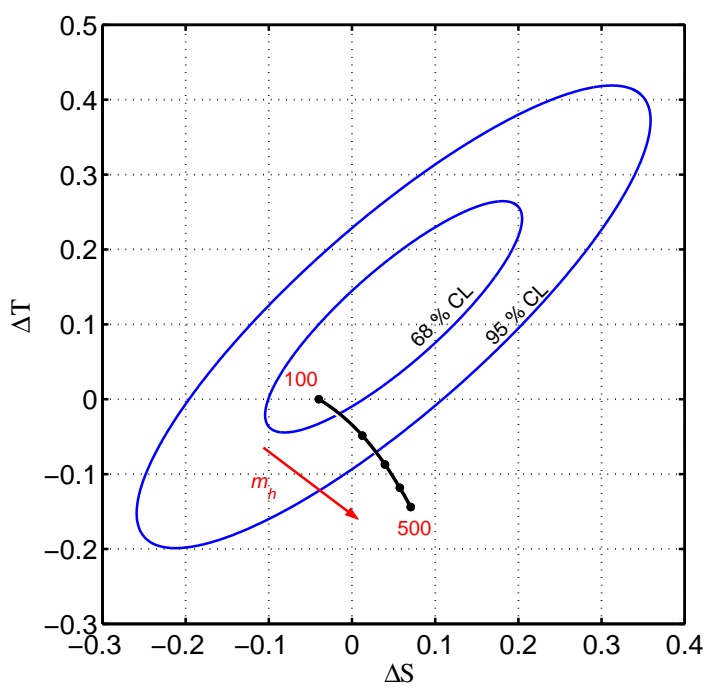

Figure 1: Experimentally allowed range for the $S$ and $T$ parameters (blue ellipses), from Ref. [1]. The $\Delta S=\Delta T=0$ point corresponds to the SM prediction for $m_{t}=175 \mathrm{GeV}$ and $m_{h}=100 \mathrm{GeV}$. The black curve is the $\mathrm{SM}$ prediction for $m_{t}=171.4 \mathrm{GeV}$ and different values of $m_{h}$ (in $\mathrm{GeV}$ ).

Since $m_{h}$ (or the dimension-2 operator in $\mathscr{L}_{\text {Higgs }}$ ) is quadratically sensitive to the cut-off, the hierarchy between $\Lambda$ and $m_{h}$ poses a naturalness problem to the theory (the so-called little hierarchy problem).

\section{Breaking the electroweak symmetry without the Higgs}

If we assume that the electroweak symmetry is broken because of an underlying spontaneous breaking of a global symmetry, the low-energy dynamics of the system is universally determined by the properties of the corresponding Goldstone bosons [2]. If we assume that the underlying symmetry is $S U(2)_{L} \times S U(2)_{R} \rightarrow S U(2)_{L+R}$, as in the standard case, the bosonic part of the lowenergy effective Lagrangian has a unique operator:

$$
\mathscr{L}_{\chi}^{(2)}=\frac{v^{2}}{4} \operatorname{Tr}\left(D_{\mu} U^{\dagger} D_{\mu} U\right)
$$

where $U$ is the unitary matrix encoding the three Goldstone bosons fields $\left(\pi_{i}\right)$ :

$$
U=e^{2 i \hat{\pi} / v}, \quad \hat{\pi}=T^{a} \pi^{a}=\frac{1}{\sqrt{2}}\left[\begin{array}{cc}
\frac{\pi^{0}}{\sqrt{2}} & \pi^{+} \\
\pi^{-} & -\frac{\pi^{0}}{\sqrt{2}}
\end{array}\right], \quad U \rightarrow g_{L} U g_{R} .
$$

Not surprisingly, we obtain $\mathscr{L}_{\chi}^{(2)}$ also from $\mathscr{L}_{\text {Higgs }}$ in the limit $m_{h} \rightarrow \infty$, as it can easily be check from (2.2) re-writing $H$ as

$$
H=(v+h) \times U .
$$

In principle, to break the electroweak symmetry we do not need to assume an underlying $S U(2)_{L} \times$ $S U(2)_{R}$ global symmetry: a $S U(2)_{L} \times U(1)_{R}$ group would be sufficient. However, doing so the 
universal $\mathscr{O}\left(p^{2}\right)$ Lagrangian of the Goldstone bosons would contain also the custodial-symmetrybreaking operator

$$
O_{T}^{(2)}=\frac{v^{2}}{4}\left[\operatorname{Tr}\left(T_{3} U^{\dagger} D_{\mu} U\right)\right]^{2} .
$$

Since this operator breaks the relation between $W$ and $Z$ masses in (2.5) it must have an unnaturally small coefficient. This is why a global $S U(2)_{L} \times S U(2)_{R}$ symmetry (or any larger group containing $\left.S U(2)_{L} \times S U(2)_{R}\right)$ is the natural starting point for an effective description of the electroweak symmetry breaking mechanism.

The effective Lagrangian

$$
\mathscr{L}_{\text {eff }}^{\text {Univ }}=\mathscr{L}_{\text {gauge }}^{\mathrm{SM}}+\mathscr{L}_{\chi}^{(2)}+\mathscr{L}_{\text {Yukawa }}(H \rightarrow v \times U)
$$

provides an excellent description of particle physics, beyond the tree level, at energies below the ultraviolet cut-off

$$
\Lambda_{\chi}=4 \pi v \approx 3 \mathrm{TeV}
$$

(the naïve dimensional cut-off of $\mathscr{L}_{\chi}^{(2)}$ ). Most low-energy precision observables (including the anomalous magnetic moment of the muon, $B \rightarrow X_{s} \gamma, \Delta M_{B}$, etc...) can be computed to high precision with this effective Lagrangian and are in agreement with experimental data. There are only two potential problems, which are usually advocated as clues of new degrees of freedom below the cut-off $\Lambda_{\chi}$ :

- the violation of unitary in $W W$ scattering, if evaluated at the tree-level with $\mathscr{L}_{\text {eff }}^{\text {Univ }}$;

- the bad agreement with data of the electroweak observables $S$ and $T$, if evaluated at the one-loop level with $\mathscr{L}_{\text {eff }}^{\text {Univ }}$, using $\Lambda_{\chi}$ as ultraviolet cut-off.

The tree-level violation of unitary in $W W$ scattering cannot be considered a clear indication of new degrees of freedom below the scale $\Lambda_{\chi}$. We find exactly the same problem in $\pi \pi$ scattering within QCD. As in the QCD case, the problem could be solved by strongly interacting dynamics around the naïve ultraviolet cut-off of the effective theory. Only if the ultraviolet completion of the theory is weakly interacting, we are forced to include light degrees of freedom below $\Lambda_{\chi}$. This is what happens with the standard Higgs mechanism, where the new degrees of freedom is the physical Higgs field.

The problem of $S$ and $T$ is the most difficult one to be solved. $\mathscr{L}_{\text {eff }}^{\text {Univ }}$ allows us to evaluate the universal infrared contribution to $S$ and $T$ generated by Goldstone-boson loops. Contrary to the flavour observables mention above, this contribution is not finite. At the one-loop level one has

$$
\left.\Delta T\right|_{\mathrm{IR}}=-\frac{3}{8 \pi c_{W}^{2}} \ln \left(\frac{\Lambda}{m_{W}}\right)+\ldots,\left.\quad \Delta S\right|_{\mathrm{IR}}=\frac{1}{6 \pi} \ln \left(\frac{\Lambda}{m_{W}}\right)+\ldots
$$

where $\Lambda$ is an ultraviolet regulator and the dots are tiny finite corrections. Replacing $\Lambda$ with $\Lambda_{\chi}$ leads to a bad description of experimental data. This can be understood by noting that if we replace $\Lambda$ with $m_{h}$ we obtain, to a good accuracy, the SM contribution shown in Fig. 1 as a function of $m_{h}$. Additional contributions to $S$ and $T$ are generated by local operators of higher order in the derivative expansion. However, such contributions are naturally subleading with respect to the 
logarhythmically-enhanced infrared terms in Eq. (3.7): the naïve size of the local terms is what we obtain from (2.10) replacing $\Lambda$ with $\Lambda_{\chi}$. In order to be consistent with data we need a mechanism to enhance the local contributions: this can easily be obtained including new states below the cut-off scale $\Lambda_{\chi}$.

\section{Light vectors in the electroweak chiral Lagrangian}

An interesting alternative to the Higgs mechanism is the wide class of Higgsless models: theories where there are no light scalar particles and the $S U(2)_{L} \times U(1)_{Y} \rightarrow U(1)_{Q}$ breaking is generated by new strong dynamics above the electroweak scale (see e.g. Ref. [3-10]). A general feature of these models is that the lightest non-standard particles are massive spin-one states. These new vectors replace, partly or completely, the Higgs boson in maintaining unitarity in $W W \rightarrow W W$ scattering [11,12], and may play a significant role also in EWPO.

The phenomenology of new massive vectors at high-energy colliders [13-15], as well as their role in electroweak observables, has been widely discussed in the recent literature. However, most of the existing analyses are based on specific dynamical assumptions, such as considering these vector states as the massive gauge bosons of a hidden local symmetry. As recently discussed in [16] (see also [17]), these assumptions may be too restrictive for generic models with strong dynamics at the $\mathrm{TeV}$ scale, and only going beyond these assumptions the sole exchange of heavy vectors can provide a successful description of EWPO. More generally, the construction of an appropriate effective theory including only SM fields and these new light states is a very efficient tool to discuss theoretical and phenomenological constraints on such states.

A simple prototype is the effective theory proposed in [16], which is based only on the following rather general assumptions:

- The new strong dynamics is invariant under a global chiral symmetry, $S U(2)_{L} \times S U(2)_{R}$, broken spontaneously into $S U(2)_{L+R}$, and under the discrete parity $P: S U(2)_{L} \leftrightarrow S U(2)_{R}$.

- A pair of vector $(V)$ and axial-vector $(A)$ states, belonging to the adjoint representation of $H$, are the only new light dynamical degrees of freedom below $\Lambda_{\chi}$.

- SM fermions couple to the new $V$ and $A$ states only via the SM gauge interactions.

Under these assumptions, the low-energy interactions of the new vector fields and the SM degrees are controlled by five parameters: two masses $\left(M_{V}, A\right)$ and three effective couplings. The latter are the couplings of the three $\mathscr{O}\left(p^{2}\right)$ operators containing at most one heavy field,

$$
\mathscr{L}_{1 V}^{(2)}=\frac{i}{2 \sqrt{2}} G_{V} \operatorname{Tr}\left(V^{\mu v}\left[u_{\mu}, u_{v}\right]\right)+\frac{1}{2 \sqrt{2}} F_{V} \operatorname{Tr}\left(V^{\mu v} f_{\mu v}^{+}\right)+\frac{1}{2 \sqrt{2}} F_{A} \operatorname{Tr}\left(A^{\mu v} f_{\mu v}^{-}\right),
$$

where

$$
f_{\mu \nu}^{ \pm}=u\left(g T^{a} W_{\mu v}^{a}\right) u^{\dagger} \pm u^{\dagger}\left(g^{\prime} T_{3} B_{\mu v}\right) u, \quad u_{\mu}=i u^{\dagger} D_{\mu} U u^{\dagger}, \quad u^{2}=U
$$

Here we have chosen to describe the new massive vectors via the irreducible antisymmetric tensor fields, $V^{\mu v}$ and $A^{\mu v}$, following the formalism proposed in Ref. [18]. This formulation has the advantage of avoiding the kinetic mixing of the new states with the Goldstone boson fields, and 
allows the most general form of interaction at $\mathscr{O}\left(p^{2}\right)$. Indeed this Lagrangian is nothing but the translation to high energies of the formalism used in [19] to describe the low-energy effects of vector-meson dominance in QCD.

The three effective couplings, $F_{V, A}$ and $G_{V}$, have dimensions of mass and, by naive dimensional analysis, are expected to be of $\mathscr{O}(v)$. In specific Higgsless models, such as the so-called 3- and 4site models, these couplings are not independent and obey the "hidden-gauge" relations $F_{V}=2 G_{V}$ and $F_{A}=0$. These relations holds in all five-dimensional deconstructed models as long as we neglect non-renormalizable terms in the bulk; however, as discussed in [17], they can be violated in more general Higgsless frameworks. The effective Lagrangian in Eq. (4.1) has the advantage of allowing the description of both hidden-gauge models and more general frameworks.

The coupling $G_{V}$, which controls the coupling of the vector state to two longitudinal SM gauge bosons, can be rather constrained if we impose perturbative unitarity up to $\Lambda_{\chi}$. Imposing this condition, $G_{V}$ has to lie in the narrow range shown in Fig. 2 (left). Note that this condition does do not pose a significant constraint on the value of $M_{V}$ : as already discussed in the previous section, the unitarity condition do not necessarily imply the existence of new light states.

A more constrained picture is obtained if we require that the sole exchange of the two spin-one states leads to a satisfactory description of EWPO. This can be achieved, with a proper tuning of the free parameters, if at least one vector state is relatively light [16]. As is well known, at the tree-level the $S$ parametr receives a positive local contribution

$$
\Delta S=4 \pi\left(\frac{F_{V}^{2}}{M_{V}^{2}}-\frac{F_{A}^{2}}{M_{A}^{2}}\right),
$$

which is the analog of the vector-meson contribution to the low-energy coupling $L_{10}$ in QCD [19]. This effect alone would worsen the agreement with data; however, it can possibly be compensated by a sizable (quadratically divergent) positive contribution to $T$ generated at one-loop level by the $U(1)_{Y}$ gauge interaction. This contribution is sizable, and the EWPO can be satisfied, only if $M_{V}$ is sufficiently light [see Fig. 2 (left)] and, at the same time, the effective couplings in Eq. (4.1) do not satisfy the hidden-gauge relations.

It is worth to stress that a violation of the hidden-gauge relations with a light $M_{V}$ is a non-trivial price to pay in terms of naturalness of the effective theory. The bad ultraviolet behavior implied by the violation of the hidden gauge relations makes quite unnatural to keep $M_{V}$ relatively well below the $4 \pi v$ cut-off. For comparison, we report here the effective low-energy couplings describing the dynamics of vector mesons in $\mathrm{QCD}$, rescaled to the corresponding vacuum expectaation value $\left(v \approx 246 \mathrm{GeV} \rightarrow 93 \mathrm{MeV} \equiv F_{\pi}\right):$

$$
\begin{gathered}
F_{V}^{(\rho)}=(1.66 \pm 0.01) \times v, \quad G_{V}^{(\rho)}=(0.65 \pm 0.08) \times v, \quad F_{A}^{\left(a_{1}\right)}=(1.29 \pm 0.27) \times v, \\
M_{V}^{(\rho)}=0.66 \times(4 \pi v), \quad M_{A}^{\left(a_{1}\right)}=1.05 \times(4 \pi v) .
\end{gathered}
$$

As can be seen, in this case we have sizable devitions from the hidden gauge relations; however, the vector masses are close to the naïve cut-off.

Determining the free parameters of this effective theory from data, via the direct production of the new states at colliders is a key ingredient to test the validity of this construction. The most general signature of these states is their appearance in $W W$ (or WZ) scattering. This effect is 

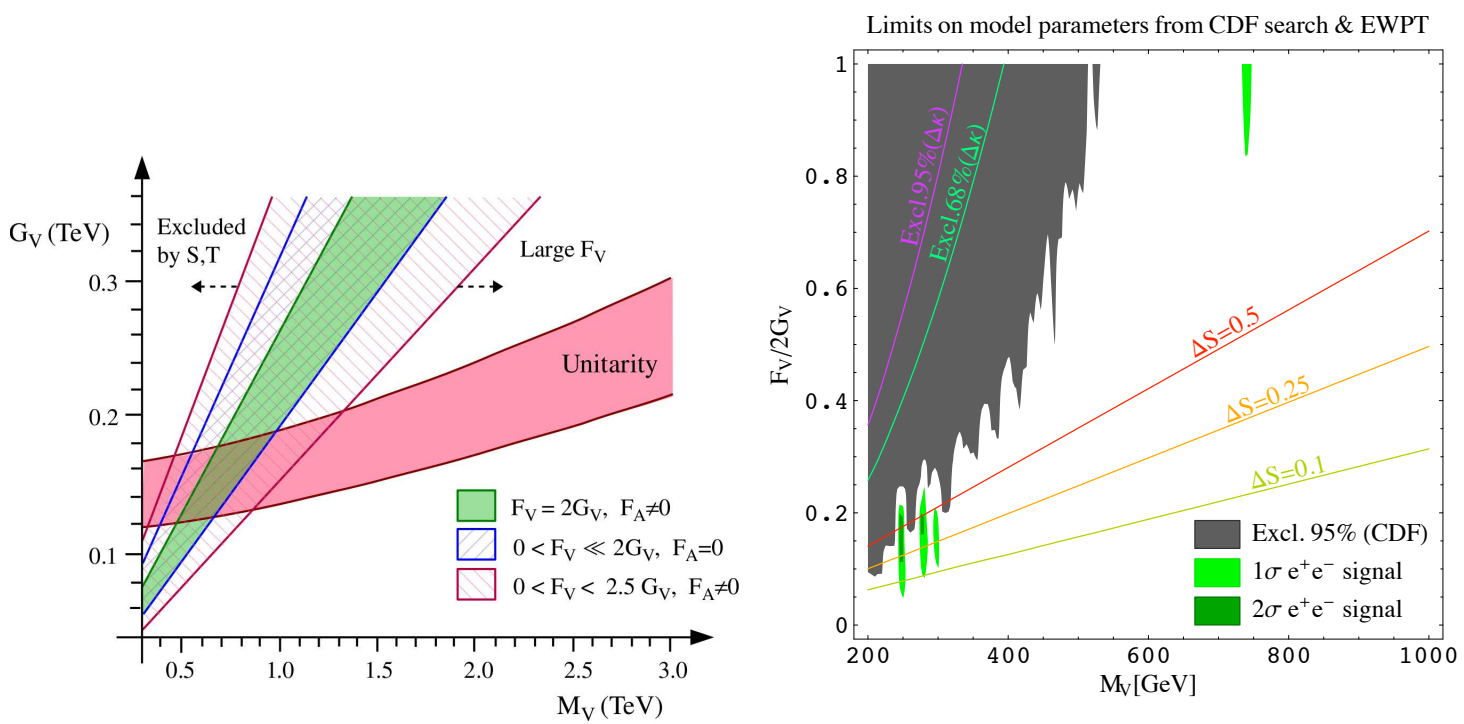

Figure 2: Left: summary of unitarity and EWPO constraints (at 95\% C.L.) in the $\left(M_{V}, G_{V}\right)$ plane [16]. Right: bounds in the $F_{V}-M_{V}$ plane from $e^{+} e^{-} \mathrm{CDF}$ data, and comparison with some of the constraints from EWPO [20].

related to the role played by the new states in unitarizing the theory and, to a large extent, it can be predicted in a model-independent way [12]. The only relevant free parameter is the mass of the lightest vector state, which is not fixed by the unitarity condition. As shown by recent analyses (see e.g. [13]), detecting such states in $W W$ scattering at the LHC is not an easy task: even for $M_{V} \sim 700 \mathrm{GeV}$ an integrated statistics of $\mathscr{O}\left(100 \mathrm{fb}^{-1}\right)$ is needed.

If the mass of at least one of the new spin-one resonances is relatively low (around or below $1 \mathrm{TeV}$ ) then such states could be detected more easily via Drell-Yan production: despite the faster drop of the signal/background ratio for rising $M_{V(A)}$, compared to $W W$ fusion, in a large fraction of the parameter space the Drell-Yan production may yield a rather large and clean non-standard signal, even for integrated statistics of $\mathscr{O}\left(1 \mathrm{fb}^{-1}\right)$ [20]. In addition to the mass spectrum, the key parameters here are the effective couplings $F_{V(A)}$, which parametrise the (gauge-invariant) mixing of the new states with the SM gauge bosons. Interestingly, the determination of these parameters could shed more light on the role of the resonances in the EWPO.

For very light masses $\left(M_{V} \lesssim 800 \mathrm{GeV}\right)$, the cleanest signal is the $\ell^{+} \ell^{-}$final state $(\ell=e, \mu)$. In this channel Tevatron is already providing significant constraints in the $F_{V}-M_{V}$ plane, as shown in Fig. 2 (right). Note that relatively low $M_{V}$ values are still allowed, provided $F_{V}$ is not maximal: a configuration which is not allowed in the simplest Higgsless models, but is possible (and even favoured by the EWPO) in a general effective theory approach. For large values of $F_{V}\left(F_{V} \approx 2 G_{V}\right)$, there are realistic chances to observe deviations from the SM at the LHC, even with a statistics of a few $\mathrm{fb}^{-1}$ [Fig. 3 (up)]. However, in this channel the signal/background ratio drops very fast with $M_{V}$ : this implies that it is almost impossible to detect a signal for $M_{V} \gtrsim 800 \mathrm{GeV}$ (even with high statistics). The $W Z$ final state could offer a wider mass reach, for sufficiently high statistics. As shown in Fig. 3 (down), the ratio between signal and irreducible background is 

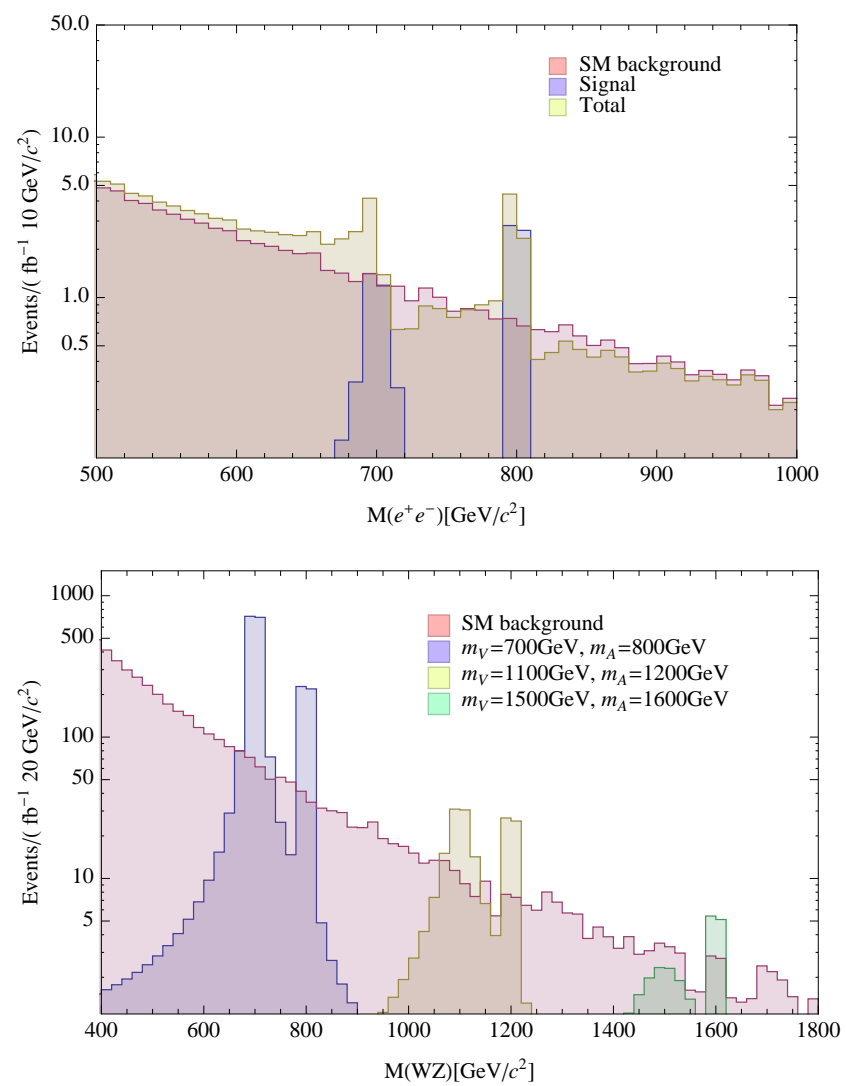

Figure 3: Possible signatures of $V$ and $A$ states in $p p \rightarrow \ell^{+} \ell^{-}$(up) or $p p \rightarrow W Z$ (down) at $\sqrt{s}=14 \mathrm{TeV}$ [20]. All resonance signals have been obtained assuming $F_{A}=F_{V}=2 G_{V}$ (condition that maximise the signal). The SM background corresponds only to the irreducible electroweak production of $\ell^{+} \ell^{-}$or $W Z$ pairs. The plots do not include the decay branching ratios of $W$ and $Z$ bosons (lower plot), as well as any experimental cut.

large even for $M_{V} \sim 1.2 \mathrm{TeV}$. Requiring leptonic decays of both $Z$ and $W$, to suppress the nonirreducible background, the mass region $M_{V} \gtrsim 1 \mathrm{TeV}$ could be explored with an integrated statistics of $\mathscr{O}\left(100 \mathrm{fb}^{-1}\right)$.

As recently dscussed in Ref. [21], another channel which could be accessile for light masses and could be useful to distinguish the different realisations of this framework is the production at the LHC of two massive vectors.

\section{The strongly-interacting light Higgs framework.}

Between the standard option of an elementary Higgs boson, and the extreme case of Higgsless theories, there is the interesting class of models where a light scalar state emerges as a pseudoGoldstone boson from a strongly-interacting framework at a scale not far from $\Lambda_{\chi}$ [22].

As we have seen in the previous section, a satisfactory description of EWPO without a light Higgs boson is possible, but it requires a non-trivial price to pay in terms of naturalness of the 
corresponding effective theory. This problem can be ameliorated if the effective theory includes a new light scalar state that, acting almost like an elementary Higgs, cuts off partially or completely the infrared-log contributions to $S$ and $T$ in Eq. (3.7). Explicit examples of models of this type are the so-called Little Higgs models [23] or the partially-composite Higgs models [24]. As pointed out in Ref. [25], at low energies we can capture the main features of all this class of models by means of an appropriate effective-theory, written in terms of the SM degrees of freedom and the new scalar field. The corresponding Lagrangian is nothing but the SM Lagrangian, with the explicit Higgs field, plus a series of higher-dimensional operators such as those shown in Eq. (2.9).

The two key parameters of this construction are $f$, the symmetry-breaking scale of the new dynamics above the electroweak scale $(\xi=v / f<1)$, and the effective coupling $g_{\rho}$, which control the mass spectrum associated with the new dynamics: beside the light scalar, the lightest nonstandard states are expected at the mass scale $m_{\rho} \sim g_{\rho} f$. These two parameters determine the suppression of the non-standard higher-dimensional operators: in the simplifying limit where the new dynamics is maximally strongly coupled $\left(g_{\rho} \sim 4 \pi\right)$, the effective scale $\Lambda$ in Eq. (2.10) can be identified with $\Lambda_{f}=4 \pi f$. For $\xi \rightarrow 0$ we recover the SM scenario (with no additional higherdimensional operators) and, not surprisingly, we find an excellent fit of EWPO with a light Higgs. However, it is clear that this is a fine-tuned limit. Even if we start with a vanishing Higgs mass at the tree-level, because the Higgs field is as Goldstone boson of the global symmetry breaking occurring at the scale $f$, radiative correction due to SM gauge interactions generate an effective Higgs mass. This is typically of order

$$
m_{h}^{2} \sim \frac{g^{2}}{16 \pi^{2}} \frac{g_{\rho}^{2}}{16 \pi^{2}} \Lambda_{f}^{2} \sim \frac{g_{\rho}^{2}}{16 \pi^{2}} \frac{(180 \mathrm{GeV})^{2}}{\xi^{2}},
$$

hence $\xi^{2}$ can be considered as a good estimator of the fine-tuning of this class of models. The situation can be improved if the new high-energy dynamics is not maximally coupled $\left(g_{\rho}<4 \pi\right)$. However, in this case local contributions to EPWO maybe parametrically be enhanced by a factor of order $16 \pi^{2} / g_{\rho}^{2}$ due to new light states (such as the vector resonances discussed in the previous section) [25]. As a result, in all cases some amount of fine-tuning cannot be avoided.

On the phenomenological side, the most interesting aspect of this construction is the appearance of an effective Higgs boson, whose couplings receive corrections of $\mathscr{O}\left(\xi^{2}\right)$ relative to their SM values. While the fine-tuned case $\xi \ll 1$ will be very hard to distinguish from the SM, there are good experimental prospects to identify this scenario if $\xi=\mathscr{O}(1)$ [26]

\section{Composite fermions}

The last option we will briefly address is the possibility of adding new fermions, with welldefined transformation properties under $S U(2)_{L+R}$, as relatively light states below the naïve cutoff $\Lambda_{\chi}$. In the two frameworks discussed before we assumed that the SM fermions were coupled to the new dynamics in a weak and flavour-universal way, only via gauge interactions. This safe assuption (as far as flavour physics is concerned) is questionable if we add new light fermion states which can mix with SM fermions. On the one hand, this mixing opens the possibility of new potentially large custodial symmetry breaking terms (similarly to the top-quark mass), which could allow a good $S-T$ fit with no or heavy Higgs field. On the other hand, this mixing leads to potential 
problems with other precision measurements, such as the $Z \rightarrow b \bar{b}$ coupling [27], the universality of charged-current interactions $[29,30]$ and various flavour-changing neutral-current (FCNC) observables [28]. A natural way to deal with these problems is to enlarge the symmetry group, possibly including some protective flavour symmetry, and to assume that the enlarged symmetry group is broken only along specific directions (see e.g. Ref. [31,32]).

As far as flavour-changing processes are concerned, the most protective assumption is the so-called MFV hypothesis. Within the quark sector, this implies that the global quark-flavour symmetry of the SM gauge Lagrangian, $\mathscr{G}_{q}=S U(3)_{Q_{L}} \times S U(3)_{u_{R}} \times S U(3)_{d_{R}}$, is broken only by two spurions (the Yukawa couplings) transforming as $Y_{U} \sim(\overline{3}, 3,1)$ and $Y_{D} \sim(\overline{3}, 1,3)$ under $\mathscr{G}_{q}$ [31]. As recently discussed in [33], a MFV structure can be implemented if we assume that the new fermions are $S U(2)_{L+R}$ singlets. However, in such case the contribtuion to $T$ is negligible. The impact in EWPO is potentially larger in the case of $S U(2)_{L+R}$ dublets or triplets [33]; however, in such case the maximal flavour symmetry group is smaller than $\mathscr{G}_{q}$ and we are left with fine-tuning problems in FCNC observables.

\section{Conclusions: the unavoidable fine-tuning problem}

As we illustrated with various examples, our knowledge about the ultraviolet completion of the effective Lagrangian in (3.5), or the SM with no Higgs, is still quite poor.

On the one hand, we have the class of models with a light Higgs field and relatively heavy new dynamics, whose extreme case is SM itself. In these models it is natural to obtain a good fit to electroweak precision observabels. However, there is an unavoidable fine-tuning problem in keeping the Higgs mass light. To some extent, this statement remains true even if the Higgs is a pseudo-Goldstone boson, or a partially composite state.

On the other hand, we have the class of models with no or heavy Higgs field. Here the finetuning problem on the Higgs mass is absent by construction; however, a naturalness problem reappear in the conditions we need to impose on the free parameters of the effective theory in order to fit the electroweak precision observabels.

At present, comparing these two types of naturalness problems is more a question of taste than a well-defined physical question. Hopefully, the problem will soon have a definite experimental answer with the direct exploration of the TeV energy range at the LHC.

\section{References}

[1] R. Barbieri, arXiv:0706.0684 [hep-ph].

[2] S. R. Coleman, J. Wess and B. Zumino, Phys. Rev. 177 (1969) 2239; C. G. . Callan, S. R. Coleman, J. Wess and B. Zumino, Phys. Rev. 177 (1969) 2247.

[3] R. Casalbuoni, S. De Curtis, D. Dominici and R. Gatto, Phys. Lett. B 155 (1985) 95; Nucl. Phys. B 282 (1987) 235.

[4] C. Csaki et al., Phys. Rev. D 69, 055006 (2004) [arXiv:hep-ph/0305237]; Phys. Rev. Lett. 92 (2004) 101802 [arXiv:hep-ph/0308038].

[5] Y. Nomura, JHEP 0311 (2003) 050 [arXiv:hep-ph/0309189]. 
[6] R. Barbieri, A. Pomarol and R. Rattazzi, Phys. Lett. B 591 (2004) 141 [arXiv:hep-ph/0310285].

[7] R. Foadi, S. Gopalakrishna and C. Schmidt, JHEP 0403 (2004) 042 [arXiv:hep-ph/0312324].

[8] R. S. Chivukula, et al., Phys. Rev. D 70 (2004) 075008 [arXiv:hep-ph/0406077].

[9] H. Georgi, Phys. Rev. D 71 (2005) 015016 [arXiv:hep-ph/0408067].

[10] R. Foadi, M. T. Frandsen, T. A. Ryttov and F. Sannino, Phys. Rev. D 76 (2007) 055005 [arXiv:0706.1696 [hep-ph]].

[11] R. S. Chivukula, D. A. Dicus and H. J. He, Phys. Lett. B 525 (2002) 175 [arXiv:hep-ph/0111016]; R. S. Chivukula and H. J. He, Phys. Lett. B 532 (2002) 121 [arXiv:hep-ph/0201164]; R. S. Chivukula, D. A. Dicus, H. J. He and S. Nandi, Phys. Lett. B 562 (2003) 109 [arXiv:hep-ph/0302263].

[12] J. Bagger et al., Phys. Rev. D 49 (1994) 1246 [arXiv:hep-ph/9306256].

[13] H. J. He et al., Phys. Rev. D 78 (2008) 031701 [arXiv:0708.2588 [hep-ph]].

[14] E. Accomando, S. De Curtis, D. Dominici and L. Fedeli, arXiv:0807.5051 [hep-ph]; Nuovo Cim. 123B (2008) 809 [arXiv:0807.2951 [hep-ph]].

[15] A. Belyaev et al., arXiv:0809.0793 [hep-ph].

[16] R. Barbieri, G. Isidori, V. S. Rychkov and E. Trincherini, Phys. Rev. D 78 (2008) 036012 [arXiv:0806.1624 [hep-ph]].

[17] J. Hirn, A. Martin and V. Sanz, JHEP 0805 (2008) 084 [arXiv:0712.3783 [hep-ph]]; Phys. Rev. D 78 (2008) 075026 [arXiv:0807.2465 [hep-ph]].

[18] G. Ecker et al., Phys. Lett. B 223 (1989) 425;

[19] G. Ecker, J. Gasser, A. Pich and E. de Rafael, Nucl. Phys. B 321 (1989) 311.

[20] O. Cata, G. Isidori and J. F. Kamenik, Nucl. Phys. B 822 (2009) 230 [arXiv:0905.0490 [hep-ph]].

[21] R. Barbieri, A. E. Carcamo, G. Corcella, R. Torre, E. Trincherini, arXiv:0911.1942

[22] D. B. Kaplan and H. Georgi, Phys. Lett. B 136 (1984) 183.

[23] N. Arkani-Hamed, A. G. Cohen, E. Katz and A. E. Nelson, JHEP 0207, 034 (2002) [arXiv:hep-ph/0206021].

[24] R. Contino, Y. Nomura and A. Pomarol, Nucl. Phys. B 671, 148 (2003) [arXiv:hep-ph/0306259]; K. Agashe, R. Contino and A. Pomarol, Nucl. Phys. B 719, 165 (2005) [arXiv:hep-ph/0412089].

[25] G. F. Giudice, C. Grojean, A. Pomarol and R. Rattazzi, JHEP 0706 (2007) 045 [arXiv:hep-ph/0703164].

[26] C. Grojean, arXiv:0910.4976 [Unknown].

[27] K. Agashe, R. Contino, L. Da Rold and A. Pomarol, Phys. Lett. B 641 (2006) 62 [arXiv:hep-ph/0605341].

[28] G. Isidori, arXiv:0908.0404 [hep-ph].

[29] M. Antonelli et al. [FlaviaNet Working Group on Kaon Decays], arXiv:0801.1817 [hep-ph].

[30] V. Bernard, M. Oertel, E. Passemar and J. Stern, JHEP 0801 (2008) 015 [arXiv:0707.4194 [hep-ph]].

[31] G. D’Ambrosio, G. F. Giudice, G. Isidori and A. Strumia, Nucl. Phys. B 645 (2002) 155 [arXiv:hep-ph/0207036].

[32] J. Hirn and J. Stern, Eur. Phys. J. C 34 (2004) 447 [arXiv:hep-ph/0401032].

[33] R. Barbieri, G. Isidori and D. Pappadopulo, JHEP 0902 (2009) 029 [arXiv:0811.2888 [hep-ph]]. 